\title{
Ultrahighly Efficient X-ray Detection System Of Two Very Large Sized SDDs for Aberration Corrected 300 kV Microscope
}

\author{
Ichiro Ohnishi $^{1}$, Kouji Miyatake ${ }^{1}$, Yu Jimbo $^{1}$, Yorinobu Iwasawa ${ }^{1}$, Masaki Morita ${ }^{1}$, Takeo Sasaki ${ }^{1}$, \\ Hidetaka Sawada ${ }^{1}$, and Eiji Okunishi ${ }^{1}$ \\ 1. JEOL Ltd., EM Business Unit, 3-1-2 Musashino, Akishima, Tokyo, Japan
}

Analytical capability with an energy-dispersive X-ray spectroscopy (EDS) in a transmission electron microscope (TEM) has been dramatically improved by using recent silicon drift detector (SDD) technology. High flexibility for the size and shape of sensor allows the SDD with large-sized sensor to be set in an optimized configuration around an objective lens pole-piece for increasing X-ray detection efficiency.

JEM-ARM300F is our latest aberration corrected $300 \mathrm{kV} \mathrm{S} / \mathrm{TEM}$ with a cold field emission electron gun. Unique X-ray detecting system for JEM-ARM300F has been developed by the combination of a newly designed wide gap pole-piece (WGP) and two $100 \mathrm{~mm}^{2}$-sized SDDs (SDD1 \& SDD2) [1]. The X-ray collection angles of SDD1 and SDD2 for this system are measured to be $0.552 \mathrm{sr}$ and $1.08 \mathrm{sr}$, respectively with use of an accurate calculation, where the solid angle is calculated based on the area of projected detector shadow on a unit sphere. The calculation was performed by a computer aided design tool using the precise detector geometries (shape, distance and tilt angle). The experimental measurement using NiOx film specimen confirmed the estimated values as 0.593 sr for SDD1 and 0.994 sr for SDD2 [2].

Recently, we have refined the X-ray detecting system to have a further improved the X-ray detection efficiency. New detecting system uses two SDDs with $158 \mathrm{~mm}^{2}$-sized sensors. The configuration of SDDs in the new system is similar to the previous system, that is, SDD1 is perpendicular to specimen holder rod axis with a take-off angle of 30.45 degrees, whereas SDD2 is along with the holder rod axis with a take-off angle of 30.55 degrees. In the new system, collection angles of SDD1 (=1.106 sr) and SDD2 (=1.108 sr) are similar. The total collection angle of new system reaches more than $2 \mathrm{sr}(2.214 \mathrm{sr})$.

Analytical performance of new detecting system for JEM-ARM300F was measured by using NiOx film specimen (Ted Pella Inc.). Figure 1 shows a comparison of gross intensities of Ni Ka line for $100 \mathrm{~mm}^{2}-$ and $158 \mathrm{~mm}^{2}$-SDDs. The system is equipped in JEM-ARM300F with WGP configuration. The experiment was performed under the same analytical conditions: acceleration voltage $=300 \mathrm{kV}$, probe current $=500 \mathrm{pA}, \mathrm{X}$-ray acquisition time $=100 \mathrm{sec}$. In case of SDD2, the intensity of Ni Ka line showed similar value in $100 \mathrm{~mm}^{2}$ - and $158 \mathrm{~mm}^{2}$-SDDs. On the other hand, in case of SDD1, the intensity of $\mathrm{Ni}$ $\mathrm{K} \alpha$ line for $158 \mathrm{~mm}^{2}$-SDD showed about 2 times higher value than that for $100 \mathrm{~mm}^{2}$-SDD, since the distance between sample and detector is improved drastically for the $158 \mathrm{~mm}^{2}$-SDD1. Total intensity of SDD1+SDD2 for $158 \mathrm{~mm}^{2}$-SDDs showed about 1.3 times higher than that for $100 \mathrm{~mm}^{2}$-SDDs. These results accord well with the difference of total collection angle between $158 \mathrm{~mm}^{2}-(2.214 \mathrm{sr})$ and 100 $\mathrm{mm}^{2}$-SDDs (1.632 sr) systems.

Figure 2 shows atomic resolution X-ray maps for $\mathrm{SrTiO}_{3}$ [100] by using the new detecting system. Acceleration voltage was $80 \mathrm{kV}$. Mapping size was 1024 x 1024 pixels. Probe current was approximately 32 pA. X-ray acquisition time was about 10 minutes. Because of high definition maps, 
we can recognize each atomic column even when we enlarge the maps. Our new X-ray detecting system for JEM-ARM300F enables us to take such a high definition maps in a realistic acquisition time.

Our new X-ray detecting system using two SDDs with $158 \mathrm{~mm}^{2}$-sized sensors for JEM-ARM300F can provide a further high X-ray collection ability. Thus, since the system could allow us to take a chemical analysis with a shorter acquisition time than before, it can probably help us to analyze high electron beam sensitive materials such as polymer, catalysis, zeolite, and so on.

\section{References:}

[1] T. Sasaki et al, Micros. Microanal. 21. Suppl. 3 (2015) p.1861-1867.

[2] M. Watanabe et al, Micros. Microanal. 21. Suppl. 3 (2015) p.1223-1224.

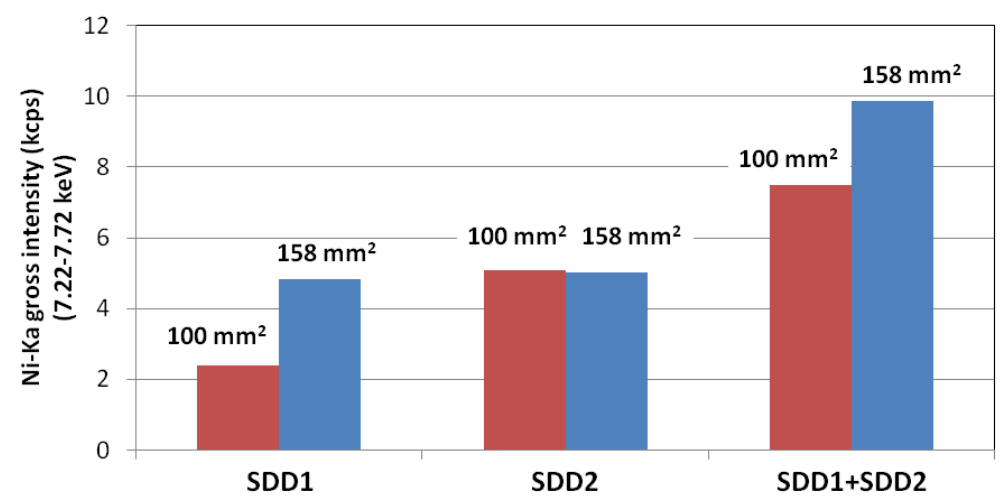

Figure 1. Comparison of gross intensity of $\mathrm{Ni} \mathrm{K \alpha}$ line (kcps) between $100 \mathrm{~mm}^{2}$ SDDs system and $158 \mathrm{~mm}^{2}$ SDDs system under the same analytical condition. Acceleration voltage was $300 \mathrm{kV}$. Probe current was 500 pA. X-ray acquisition time was 100 seconds.
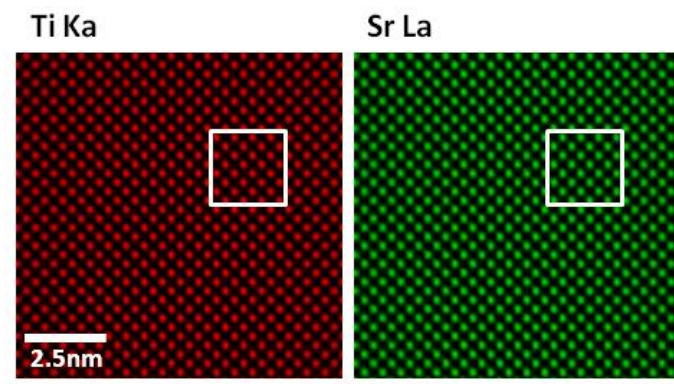

Overlay
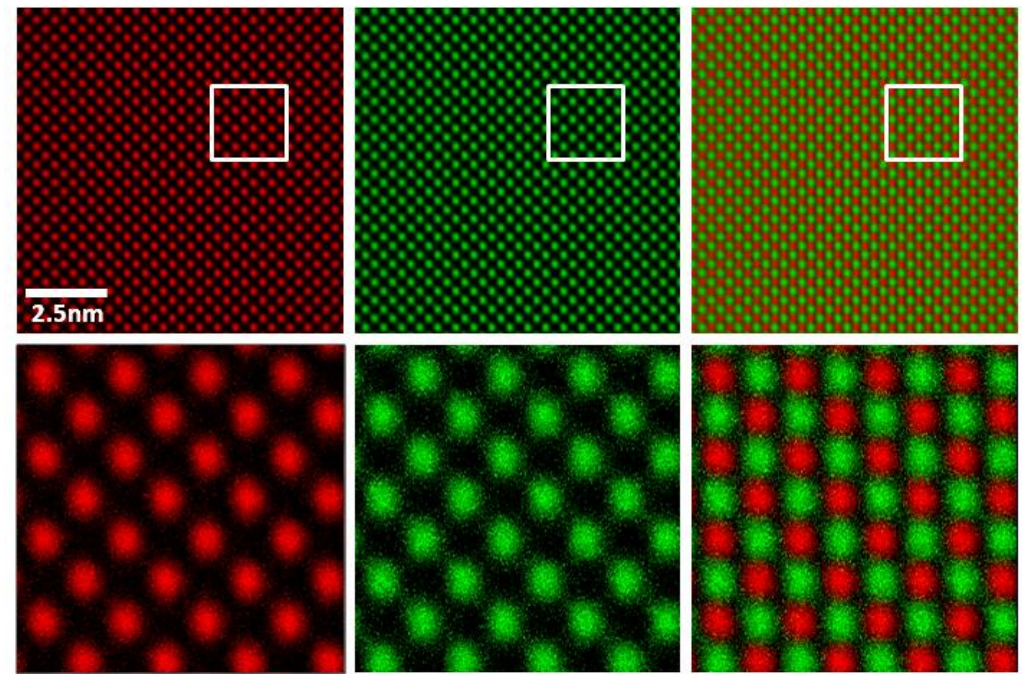

Figure 2. EDS elemental maps (Wiener filtered) of $\mathrm{SrTiO}_{3}[100]$ taken at $80 \mathrm{kV}$ with a probe current of $32 \mathrm{pA}$, a convergence semi-angle of $24 \mathrm{mrad}$, a map size of 1024 x 1024 pixels and acquisition time of 10 minutes. Lower images are enlarged images of boxed area in upper images. 